\title{
Research on Operational Decision Scheme Evaluation Method
}

\author{
Guo Lei Sun ${ }^{\mathrm{a}}{ }^{*}$, Dong Li ${ }^{\mathrm{b}}$, Xin Qi Guo ${ }^{\mathrm{c}}$ \\ Naval Aeronautical and Astronautical University, Yantai, China \\ azishisun@163.com, bidong501@sohu.com, chyguoxq@163.com
}

Keywords: Operational decision plan; Interval numbers; Attribute weights; Grey relation projection method; Evaluate.

\begin{abstract}
Operational decision scheme evaluation is an important means of inspection that operations are determined accurately or not. This article explores the evaluation methods of combat determination method, and proposes a decision scheme evaluation method based on interval numbers grey relation projection. This article introduces the basic concept and basic principle of the method, established a decision-making model which justify the decision plan's coordinate with positive ideal solution or the negative one.
\end{abstract}

\section{Introduction}

Program evaluation on operational resolution, that is to make comprehensive assessment of the feasibility, works as an important means of inspection on the accuracy of operational resolution and plays an important role in decision-making and decision-making efficiency [1,2]. The research on Operational decision-making scheme evaluation and sorting [3], essentially a multi-objective decision problem has not yet formed a system with a broad range of instructional theory [4], nor a universally recognized ideal evaluation method.

To evaluate operational determination scheme, the probability of pure analytic calculation is very difficult to cover the uncertain factors affecting operational decisions. Interval number and fuzzy number can well reflect the uncertainty of parameters, the parameter values in a variety of types, such as precision number, interval number and fuzzy number) in the form of the decision matrix which constitutes the mixed problem of the multi-target appraisal. This article discusses evaluation problem from the uncertainty of type of index weight and fuzzy number of hybrid multi-index respect to do numerical, interval number. This paper proposes a scheme evaluation method based on interval number grey relation projection for the scheme evaluation problem provides a new solution.

\section{The principle and the evaluation model of interval number grey relation projection based on Effectiveness}

Scheme evaluation and sorting problem is the selection of decision makers in the face of multiple solutions, the use of two or more than two standard (i.e. indicators or attributes) on the comprehensive evaluation of each scheme and the priorities of the scheme and choose satisfactory solution.

Assumed the solution sets as $S=\left\{S_{1}, S_{2}, \ldots, S_{m}\right\}$ and the attribute sets( cited as index set such as "cost type" and "benefit type" ) as $P=\left\{P_{1}, P_{2}, \ldots, P_{n}\right\}$.weight vector $\left(w=\left\{w_{1}, w_{2}, \ldots, w_{n}\right\}\right)$ donates the status of properties, in which the weighting equal to $w_{j}=\left[w_{j}{ }^{L}, w_{j}^{R}\right], \quad \sum_{j=1}^{n} w_{j}{ }^{L} \leq 1, \sum_{j=1}^{n} w_{j}^{R} \geq 1, \quad 0 \leq w_{j}{ }^{L} \leq w_{j}{ }^{R} \leq 1$, $j=1,2, \ldots, n$.The following are other variates.

$x_{i j}$ denotes the value of set $S_{i}$ in the attribute sets $P_{j}$ which can be a variety of types (such as precision number, interval number and fuzzy number) of the form, thus come to constitute a hybrid original evaluation matrix say $X=\left(x_{i j}\right)_{m \times n}, \quad i=1,2, \ldots, m, \quad j=1,2, \ldots, n$, which formulated as 


$$
x_{i j}= \begin{cases}x_{i j}, & \text { valules are precision number } \\ {\left[x_{i j}^{L}, x_{i j}^{R}\right],} & \text { valules are interval number } \\ {\left[x_{i j}^{L}, x_{i j}^{M}, x_{i j}^{U}\right],} & \text { valules are fuzzy number }\end{cases}
$$

Initial evaluation matrix forms the interval number. The precise number and triangular fuzzy Numbers is transformed into interval number to form the interval number initial evaluation matrix $Y=\left(\left[y_{i j}^{L}, y_{i j}^{R}\right]\right)_{m \times n}$ :

$$
\left[y_{i j}^{L}, y_{i j}^{R}\right]= \begin{cases}{\left[x_{i j}, x_{i j}\right],} & \text { valules are precision number } \\ {\left[x_{i j}^{L}, x_{i j}^{R}\right],} & \text { valules are interval number } \\ {\left[\left(x_{i j}^{L}+x_{i j}^{M}\right) / 2,\left(x_{i j}^{M}+x_{i j}^{U}\right) / 2\right],} & \text { valules are fuzzy number }\end{cases}
$$

Construct the positive and negative ideal interval number of initial evaluation matrix. Assume the index of an ideal solution $S_{0}^{+}$as $\left[y_{0 j}^{L^{+}}, y_{0 j}^{R+}\right](j=1,2, \ldots, n)$, and satisfy the following circumstance:

$$
\begin{aligned}
& {\left[y_{0 j}^{L+}, y_{0 j}^{R+}\right]=\left[\max \left(y_{1 j}^{L}, \ldots, y_{m j}^{L}\right), \max \left(y_{1 j}^{R}, \ldots, y_{m j}^{R},\right)\right]} \\
& P_{j} \text { as cost type } \\
& {\left[y_{0_{j}}^{L+}, y_{0_{j}}^{R+}\right]=\left[\min \left(y_{1 j}^{L}, \ldots, y_{m j}^{L}\right), \min \left(y_{1 j}^{R}, \ldots, y_{m j}^{R},\right)\right]}
\end{aligned}
$$

Augmented matrix type $Y^{+}=\left(\left[y_{i j}^{L+}, y_{i j}^{R+}\right]\right)_{(m+1) \times n}(i=0,1, \ldots, m ; j=1,2, \ldots, n)$ turn out be solution set of index set which is ideal interval number of initial evaluation matrix. Therefore, solution set or $S$ is related to $P$ as followed:

$$
Y^{-}=\left(\left[y_{i j}^{L-}, y_{i j}^{R-}\right]\right)_{(m+1) \times n}(i=0,1, \ldots, m ; j=1,2, \ldots, n)
$$

Construct the positive and negative ideal interval evaluation matrix. To align the ideal interval number of initial value as the followed to get normalized matrix as the ideal interval number of matrix:

$$
Y^{+}=\left(\left[y_{i j}^{L+}, y_{i j}^{R+}\right]\right)_{(m+1) \times n}(i=0,1, \ldots, m ; j=1,2, \ldots, n) \quad Z^{+}=\left(\left[z_{i j}^{L^{+}}, z_{i j}^{R+}\right]\right)_{(m+1) \times n}
$$

When $P_{j}$ is benefit type:

$$
\left[z_{i j}^{L+}, z_{i j}^{R+}\right]=\left[\frac{y_{i j}^{L+}}{y_{0 j}^{L+}}, \frac{y_{i j}^{R+}}{y_{0 j}^{R+}}\right]
$$

When $P_{j}$ is cost type:

$$
\left[z_{i j}^{L+}, z_{i j}^{R+}\right]=\left[\frac{y_{0 j}^{L+}}{y_{i j}^{L+}}, \frac{y_{0 j}^{R+}}{y_{i j}^{R+}}\right]
$$

Then the negative ideal interval evaluation matrix: $Z^{-}=\left(\left[z_{i j}^{L-}, z_{i j}^{R-}\right]\right)_{(m+1) \times n}$ 。

According to the interval number multiplication formula, calculate the positive and negative ideal interval number of weighted evaluation matrix $Z^{+}$and $Z^{-}$:

$$
\begin{aligned}
& Z^{+}=\left(\left[z_{i j}^{L+}, z_{i j}^{R+}\right]\right)_{(m+1) \times n}=\left(\left[w_{j}^{L}, w_{j}^{R}\right]\left[\left[z_{i j}^{L+}, z_{i j}^{R+}\right]\right)_{(m+1) \times n}\right. \\
& Z^{-}=\left(\left[z_{i j}^{L-}, z_{i j}^{R-}\right]\right)_{(m+1) \times n}=\left(\left[w_{j}^{L}, w_{j}^{R}\right]\left[\left[z_{i j}^{L-}, z_{i j}^{R-}\right]\right)_{(m+1) \times n}\right.
\end{aligned}
$$


To construct the positive and negative ideal grey relational evaluation matrix. The row vector in $Z^{+}=\left(\left[z_{i j}^{L+}, z_{i j}^{R+}\right]\right)_{(m+1) \times n}$ and that in $S_{0}^{+}$work out to get ideal grey relational evaluation matrix or $G^{+}=\left(r_{i j}^{+}\right)_{(m+1) \times n}, \quad i=0,1, \ldots, m ; j=1,2, \ldots, n$, in case:

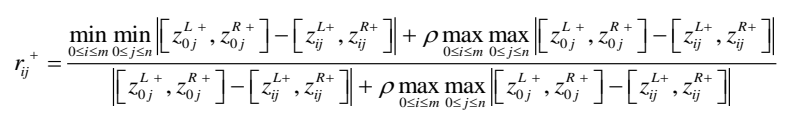

The interval distance calculation chosen is geometric distance formula in this paper $[7,8]$ :

$$
\left|\left[z_{0 j}^{L+}, z_{0 j}^{R+}\right]-\left[z_{i j}^{L+}, z_{i j}^{R+}\right]\right|=\left(\left(z_{0 j}^{L+}-z_{i j}^{L+}\right)^{2}+\left(z_{0 j}^{R+}-z_{i j}^{R+}\right)^{2}\right)^{1 / 2}
$$

$r_{0 j}{ }^{+}=1, \rho$ as resolution ratio, $\rho \in[0,1]$, assumed to be $\rho=0.5$. The negative ideal grey relational evaluation matrix can be defined as $G^{-}=\left(r_{i j}^{-}\right)_{(m+1) \times n}, i=0,1, \ldots, m ; j=1,2, \ldots, n$.

Grey relation projection principle. Assuming each decision scheme as a row vector, $\theta_{i}$ (the grey relation projection Angle) is formed between $S_{i}$ (Decision Solutions) and $S^{*}$ (ideal one) and the cos is calculated as:

$$
\cos \theta_{i}=\frac{S_{i}\left\lceil S^{*}\right.}{\left\|S_{i}\right\|\left\|S^{*}\right\|}=\frac{\sum_{j=1}^{n} r_{i j} r_{0 j}}{\sqrt{\sum_{j=1}^{n} r_{i j}{ }^{2}} \square \sqrt{\sum_{j=1}^{n} r_{0 j}{ }^{2}}}
$$

In Eq.13, $i=1,2, \ldots, m$, with the $\theta_{i}$ reduce, $\cos \theta_{k}$ grows as $\theta_{i}$ declines, indicating the consistent change direction between $S_{i}$ and $S^{*}$.

Assuming the modulus of $S_{i}$ is $d_{i}=\sqrt{\sum_{j=1}^{n} r_{i j}^{2}}$, close degree of each scheme and ideal scheme can be reflected taking the variation of modulus $\left(d_{i}\right)$ and $\cos \theta_{i}$ into consideration.

The grey relation projection $D_{i}=d_{i} \llbracket \cos \theta_{i}$ is the projection of decision scheme $S_{i}$ at ideal scheme $S^{*}$. The decision schemes values $S_{i}$ on grey relation projection for positive ideal solution $D_{i}^{+}$and negative ideal solution on the grey relation projection values $D_{i}^{-}$are as following:

$$
\begin{aligned}
& D_{i}^{+}=\frac{\sum_{j=1}^{n} r_{r_{j}{ }^{+} r_{0 j}{ }^{+}}}{\sqrt{\sum_{j=1}^{n}\left(r_{0 j}^{+}\right)^{2}}}, \quad i=1,2, \ldots, m \\
& D_{i}^{-}=\frac{\sum_{j=1}^{n} r_{r_{j}-} r_{0 j}^{-}}{\sqrt{\sum_{j=1}^{n}\left(r_{0 j}^{-}\right)^{2}}}, \quad i=1,2, \ldots, m
\end{aligned}
$$

For decision scheme $S_{i}, D_{i}^{+}$is the bigger the better, and $D_{i}^{-}$is as small as better.

Grey relation projection coefficient. In sort of scheme $S_{i}$, hoping for the best plan is closest to the ideal solution, and also the most far away from the negative ideal solution. $E_{i}$ is defined as grey relation projection coefficient to comprehensive measure scheme $S_{i}$ close to the positive ideal solution and away from negative ideal solution.

Theorem 1[9]: For scheme $S_{i}$, assuming that the grey relation projection values of positive and negative ideal solution are $D_{i}^{+}$and $D_{i}^{-}$, the grey relation projection coefficient as

$$
E_{i}=\frac{D_{i}^{+2}}{D_{i}^{+2}+D_{i}^{-2}}
$$

Sort grey relation projection coefficient value $E_{i}$ from big to small, the maximum value $E_{i}$ is the 
optimal evaluation schemes.

\section{Evaluation algorithms}

To sum up the theory and model, based on the interval grey relation projection method of effectiveness evaluation steps can be summarized as follows:

Step 1 According to the known solution set $S$ and index set $P$, construct mixed initial evaluation matrix $X$.

Step 2 Evaluation index into precise Numbers and triangle fuzzy Numbers is transformed into interval number, the formation of interval number $Y$ initial evaluation matrix 。

Step 3 To construct ideal interval number of initial evaluation matrix $Y^{+}$and the negative ideal interval number of initial evaluation matrix $Y^{-}$.

Step 4 Initial treatment of the positive, negative ideal interval number evaluation matrix $Y^{+}$and $Y^{-}$, and obtained positive, negative ideal interval number evaluation matrix $Z^{+}$and $Z^{-}$; According to the interval numbers multiplication formula[10], calculation of the positive, negative ideal interval number evaluation matrix and weight.

Step 5 All the row vector of the positive (negative) ideal interval number weighted evaluation matrix $Z^{+}$and $Z^{-}$respectively compared to the index row vector of positive (negative) scheme $S_{0}^{+}$

$\left(S_{0}^{-}\right)$calculation of grey relation coefficient, thus forming positive, negative ideal grey relational evaluation matrix $G^{+}$and $G^{-}$.

Step 6 Calculation the value $D_{i}^{+}$and $D_{i}^{-}$.of grey relation projection for every scheme $S_{i}$ for the positive and negative ideal solution.

Step 7 Calculation coefficient of grey correlation projection of each scheme $S_{i}$.

Step 8 Accordance with the grey relation projection coefficient $E_{\mathrm{i}}$ value from big to small order; make a comprehensive and objective assessment of each program.

\section{Summary}

This paper combines the concept of grey relational degree, the concept of TOPSIS and the vector projection method, according to the operation rules of interval number, interval number is proposed of grey correlation projection method for assessment of efficacy. This method is suitable for evaluation of multi index of mixed type in weight is not completely determined (such as precision number, interval number, fuzzy number), which has broad applicability.

\section{References}

[1] Q.Y. Cheng. Operations Analysis of the Combat Command Decision (Beijing: Military Science Press, 2004). (In Chinese).

[2] Przemieniecki J S. Mathematical Methods in Defense Analyses (American institute of aeronautics and astronautics, 2000).

[3] H.B. Zhang, W. Zheng and L.K. Zhu. Journal of Astronautics. Vol. 28 (2007) No.2, p.394-397.

[4] Z.S. Xu. Systems Engineering Theory and Practice. Vol. 22 (2002) No.1, p.43-47.(In Chinese).

[5] S L T. Fuzzy Sets and Systems. Vol. 50 (1992) No.1, p.247-255.

[6] J.L. Deng. The Basic Method of Grey System(Wuhan: Huazhong University of Science and Technology press, 2005). (In Chinese).

[7] J. Wu. Systems Engineering. Vol 22 (2004) No.8, p.1-4.

[8] Y.D. Dang. Proceedings of the Eighth National Youth Management Science and System Science Conference (Nanjing, Hohai University, 2005). Vol. 1, p.1095-1099. (In Chinese). 
[9] H.F. Ke, Y.G. Chen and B. Xia. Journal of Electronic. Vol. 35 (2007) No.9, p.1757-1761. (In Chinese).

[10] J.J. Zhang. Systems Engineering and Electronic Technology. Vol. 27 (2005) No.6, p.1030-1033.(In Chinese). 